\title{
Adsorbate hopping via vibrational-mode coupling induced by femtosecond laser pulses
}

\author{
H. Ueba, ${ }^{1}$ M. Hayashi, ${ }^{1}$ M. Paulsson, ${ }^{2}$ and B. N. J. Persson ${ }^{3}$ \\ ${ }^{1}$ Department of Electronics, Graduate School of Science and Engineering, University of Toyama, Toyama 930-8555, Japan \\ ${ }^{2}$ Division of Physics, School of Pure and Applied Natural Sciences, University of Kalmar, 39182 Kalmar, Sweden \\ ${ }^{3}$ IFF, FZ-Jülich, 52425 Jülich, Germany
}

(Received 29 June 2008; revised manuscript received 5 August 2008; published 22 September 2008)

\begin{abstract}
We study the heat transfer from femtosecond laser-heated hot electrons in a metal to adsorbates in the presence of vibrational-mode coupling. The theory is successfully applied to the experimental result of atomic oxygen hopping on a vicinal $\mathrm{Pt}(111)$ surface. The effective friction coupling between hot electrons and the vibrational mode relevant to the hopping motion depends on the transient temperature of the partner mode excited by hot electrons. The calculated two-pulse correlation and fluence dependence of the hopping probability reproduce the experimental results, which were previously analyzed using the hot-electron temperature $\left(T_{e}\right)$-dependent friction $\eta_{a}\left(T_{e}\right)$ in a conventional heat transfer equation. A possible elementary process behind such a hypothetic modeling using $\eta_{a}\left(T_{e}\right)$ is discussed in terms of an indirect heating of the vibrational mode for hopping at the surface.
\end{abstract}

DOI: 10.1103/PhysRevB.78.113408

PACS number(s): 82.53.St, 68.43.Pq, 68.43.Jk

Real-time monitoring of adsorbate motions and of chemical reactions induced by femtosecond pulse laser heating of hot electrons in a metal substrate has been a major goal and challenge in surface reaction dynamics. ${ }^{1}$ Recently two different groups carried out time-resolved study of lateral motion of adsorbates on vicinal Pt surfaces using femtosecond laser pulses. ${ }^{2,3}$ Employing optical second harmonic generation spectroscopy, Stépán et $a .^{3}$ measured the hopping rate of atomic oxygen for step-terrace diffusion on a vicinal $\mathrm{Pt}(111)$ surface. The experimental results of the two-pulse correlation (2PC) and the laser fluence dependence of the hopping probability have been reproduced assuming a hot-electron temperature $\left(T_{e}\right)$-dependent friction $\eta_{a}\left(T_{e}\right)$ in the conventional heat transfer equation

$$
\frac{d T_{a}}{d t}=\eta_{a}\left[T_{e}-T_{a}\right]
$$

in order to calculate the transient adsorbate temperature $T_{a}(t)$. They proposed, as a possible mechanism behind such a hypothetic $T_{e}$-dependent friction, that it may involve a primary excitation of the O-Pt vibration by hot electrons, which then couples anharmonically to the frustrated translation (FT) mode of the $\mathrm{O}$ atom required to overcome the barrier for lateral motion. This mechanism seems to be supported by several facts. The anharmonic mode coupling in the $\mathrm{O}-\mathrm{Pt}(111)$ system has been found in the temperature dependence of the vibrational linewidth of the O-Pt stretch mode observed by infrared absorption spectroscopy. ${ }^{4}$ Indispensable role of anharmonic mode coupling has also been found in hopping of a single $\mathrm{CO}$ on $\mathrm{Pd}(110)$ (Ref. 5) and $\mathrm{NH}_{3}$ on $\mathrm{Cu}(100)$ (Ref. 6) induced by an inelastic tunneling electron with a scanning tunneling microscope. The $T_{e}$-dependent friction coupling is also a key ingredient to reproduce the 2PC and the fluence dependence for femtosecond laserinduced desorption of oxygen molecules from a $\operatorname{Pd}(111)$ surface. ${ }^{7}$ The understanding of the microscopic elementary processes, however, remains at the phenomenological level. No theoretical modeling has been developed to explain why such an anharmonic coupling leads to $T_{e}$-dependent friction. According to a theory of electronically driven adsorbate excitation, ${ }^{8}$ we do not expect $T_{e}$-dependent friction coupling to the FT mode nor to the molecule-surface stretch mode for atomic oxygen on $\mathrm{Pt}(111)$ having a broad unoccupied resonance close to the Fermi level, ${ }^{9}$ and for low-frequency vibrational modes. ${ }^{10}$

Recently we have proposed a theory for the energy transfer between adsorbates and ultrafast laser heated hot electrons $^{11}$ in the presence of the vibrational-mode coupling. ${ }^{12}$ It extends existing theories on electronic friction by considering a general anharmonic oscillator potential for the adsorbate motion and coupling between different vibrational modes of the adsorbate. The coupling to the substrate is expressed by a heat transfer coefficient that depends on the adsorbate temperature. It has been shown that the theory reduces to the conventional heat transfer Eq. (1) in the case of the harmonic oscillator and a linear electron-vibration coupling. The model has been successfully applied to describe an experiment on femtosecond laser-induced hopping of $\mathrm{CO}$ molecules on a stepped $\mathrm{Pt}(111)$ surface. ${ }^{2}$ It has been shown that even if the direct heating of the FT modes relevant for the considered reaction is too low to activate hopping, the anharmonic coupling between the FT and the frustrated rotation mode with an efficient friction coupling to the hot electron is able to heat up the FT mode in the time scale of CO hopping on $\mathrm{Pt}(553)$.

In this Brief Report the heat transfer equations for coupled harmonic oscillators are used to calculate the twopulse correlation and fluence dependence of the hopping probability of atomic oxygen on a vicinal $\mathrm{Pt}(111)$ surface. ${ }^{3}$ According to our general formula of the energy transfer between modes a (with the energy of $\left.\hbar \omega_{a}\right)$ and $\mathbf{b}\left(\hbar \omega_{b}\right)$ excited by the friction coupling $\eta_{a(b)}$ to the hot electrons, the transient temperature $T_{a}(t)$ and $T_{b}(t)$ of each mode are calculated for two harmonic oscillators coupled via $V\left(u_{a}, u_{b}\right)=\Sigma_{q} \lambda_{q}\left(b_{q}^{\dagger}\right.$ $\left.+b_{q}\right) u_{a} u_{b}$, where $\lambda_{q}$ is the coupling constant and $b_{q}^{+}\left(b_{q}\right)$ is a creation (annihilation) operator of the substrate electronic excitations with the energy $\hbar \omega_{q}$, and $u_{a}, u_{b}$ are the adsorbate 
vibrational normal mode coordinates. ${ }^{12}$ Since $\hbar \omega_{a}$ is in general different from $\hbar \omega_{b}$, we need energy exchange with the substrate in order to conserve the energy through absorption or emission of small energies of electron-hole pair excitations in the substrate. The coupled heat transfer equations between $T_{e}, T_{a}$, and $T_{b}$ are given by ${ }^{12}$

$$
\begin{aligned}
& \frac{d T_{a}(t)}{d t}=\left[\eta_{a}+\eta_{a b} \frac{k_{B} T_{b}}{\hbar \omega_{b}}\right]\left(T_{e}-T_{a}\right), \\
& \frac{d T_{b}(t)}{d t}=\left[\eta_{b}+\eta_{b a} \frac{k_{B} T_{a}}{\hbar \omega_{a}}\right]\left(T_{e}-T_{b}\right),
\end{aligned}
$$

where the indirect friction couplings have the relations $\eta_{a b}$ $=S \omega_{a}, \eta_{b a}=S \omega_{b}$, and $S=8 \pi\left[u_{a 0} u_{b 0} \bar{\lambda} \rho_{\left(\epsilon_{F}\right)}\right]^{2}$ [here $u_{a 0}$ and $u_{b 0}$ are the zero-point vibration amplitudes of mode $\mathbf{a}$ and $\mathbf{b}$, respectively, $\bar{\lambda}$ the averaged coupling constant, and $\rho_{\left(\epsilon_{F}\right)}$ the substrate density of states at the Fermi level (see Ref. 12 for more details)]. This leads to $\eta_{a b} / \eta_{b a}=\omega_{a} / \omega_{b}$. The mode coupling thus gives another source of temperature increase and may play a dominating role in the heating of mode a, responsible for a motion even when its direct heating via $\eta_{a}$ is small, as often is the case, e.g., for parallel adsorbate vibrations. It is noted here that after a long enough time the adsorbate-metal system reaches thermal equilibrium $T_{e}=T_{a}$ $=T_{b}$, as expected because of the coupling to the substrate electrons.

Equations (2) and (3) are combined with the so-called two-temperature model ${ }^{13}$ to calculate $T_{e}(t), T_{a}(t)$, and $T_{b}(t)$ for atomic oxygen on a Pt surface at $T=80 \mathrm{~K}$ \{using material parameters ${ }^{14}$ [Debye temperature $=240 \mathrm{~K}$, electronphonon coupling $=6.67 \times 10^{17} \mathrm{~W} \mathrm{~K}^{-1} \mathrm{~m}^{-3}$, electronic specific heat $=748 \mathrm{~J} \mathrm{~K}^{-2} \mathrm{~m}^{-2}$, thermal conductivity (at $77 \mathrm{~K}$ ) $\left.=71.6 \mathrm{~W} \mathrm{~K}^{-1} \mathrm{~m}^{-1}\right]$ and the laser fluence $=23 \mathrm{~J} / \mathrm{nm}^{2}$, Gaussian shape laser pulse width $=55 \mathrm{fs}$, and skin depth $=12.56 \mathrm{~nm}$ at laser wavelength of $800 \mathrm{~nm}\}$. Here we use $\hbar \omega_{a}=50 \mathrm{meV}$ and $\hbar \omega_{b}=60 \mathrm{meV}$, which, respectively, correspond to the atomic oxygen FT mode (the reaction coordinate mode for hopping) and the O-Pt stretch mode on a $\operatorname{Pt}(111)$ surface. ${ }^{15}$ We fix $1 / \eta_{b}=1$ ps estimated from the low-temperature limit of the linewidth of the O-Pt stretch mode. ${ }^{4}$

Figure 1 compares the time-dependent effective friction $\eta_{a}^{\text {eff }}(t)=\eta_{a}+\eta_{a b}\left(k_{B} T_{b} / \hbar \omega_{b}\right)$ of mode a due to a weak direct $\left(\eta_{a}=0.3 / \mathrm{ps}\right)$ and a strong indirect friction coupling $\left(\eta_{a b}\right.$ $=6.0 / \mathrm{ps})$ and of the $T_{e}$-dependent friction $\eta_{a}\left(T_{e}\right)=\eta_{a}^{0} T_{e}^{2}(t)$ with $\eta_{a}^{0}=1.8 \times 10^{5} \mathrm{~K}^{-2} \mathrm{ps}^{-13}$, where $F=23 \mathrm{~J} / \mathrm{nm}^{2}$ is used to calculate $T_{e}(t), T_{a}(t)$, and $T_{b}(t)$ from Eqs. (1)-(3). The transient behavior of $\eta_{a}^{\text {eff }}(t)$ is the same as that of $T_{b}(t)$. Because of the efficient coupling of the $\mathbf{b}$ mode to hot electrons $T_{b}(t)$ immediately follows $T_{e}(t)$. Figure 2 shows $T_{e}(t)$ and $T_{a}(t)$ after excitation with a pair of two 55 fs pulses with $F$ $=23 \mathrm{~J} / \mathrm{nm}^{2}$ fluence each and a temporal pulse delay of $3 \mathrm{ps}$. The rest of the parameters are the same as in Fig. 1.

Figure 2 shows $T_{e}(t)$ (red curve) and $T_{a}(t)$ (black) calculated for $\eta_{a}=0.3 / \mathrm{ps}$ with the mode coupling $\eta_{a b}=6.0 / \mathrm{ps}$ to the $\mathbf{b}$ mode $\left(\eta_{b}=1.0 / \mathrm{ps}\right)$, using Eqs. (2) and (3) (the fluence is $23 \mathrm{~J} / \mathrm{nm}^{2}$ ). For comparison we also plot $T_{a}(t)$ calculated from Eq. (1) with a constant friction (green curve) of $\eta_{a}$

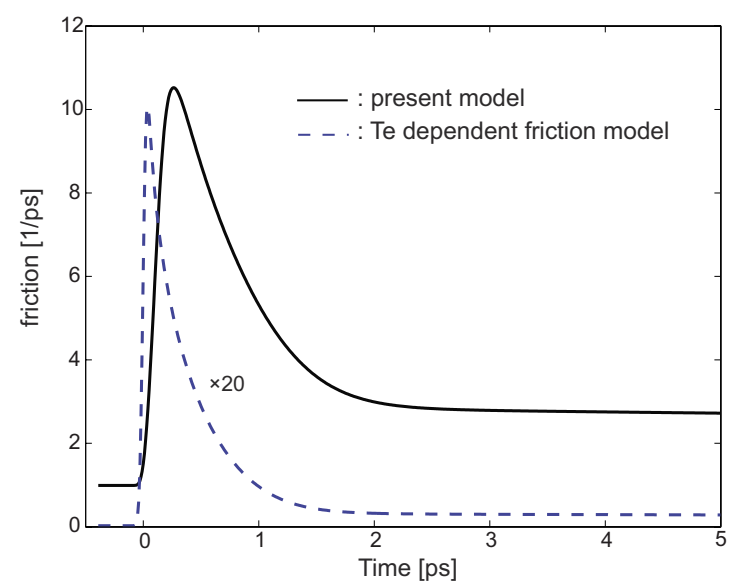

FIG. 1. (Color online). Transients of the effective friction $\eta_{a}^{\text {eff }}(t)$ (black curve) and the $T_{e}$-dependent friction $\eta_{a}\left(T_{e}\right)$ (blue-dashed curve). See the text for the parameters used herein.

$=0.3 / \mathrm{ps}$, as well as with a temperature-dependent friction (blue-dashed curve) $\eta_{a}\left(T_{e}\right)=\eta_{a}^{0} T_{e}^{2}, \eta_{e}^{0}=1.8 \times 10^{5} \mathrm{~K}^{-2} \mathrm{ps}^{-13}$. It is found that $T_{a}(t)$ can be substantially heated up to higher temperature via the mode coupling than that calculated using Eq. (1) with a constant and $T_{e}(t)$-dependent friction.

Knowing the time-dependent adsorbate temperature $T_{a}(t)$ describing the vibrational excitation of the adsorbate, the reaction rate is then given by an Arrhenius-type expression as

$$
R(t)=k_{0} e^{-U_{0} / k_{B} T_{a}(t)},
$$

where $k_{0}$ is a prefactor. Gudde et al. ${ }^{15}$ compared the transient behaviors of $T_{a}(t)$ and $R(t)$ calculated using Eqs. (1) and (4) and the generalized friction model, ${ }^{8}$

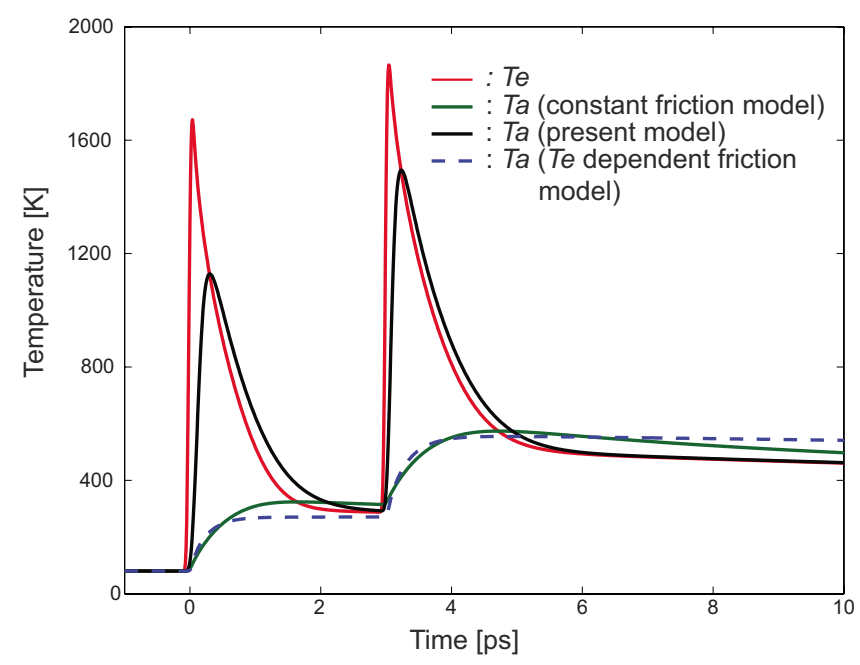

FIG. 2. (Color online). Temperature transients of the electron $T_{e}(t)$ (red curve) and a mode $T_{a}(t)$ with $\left(\eta_{a}=0.3 / \mathrm{ps}\right.$; black curve) and without $\left(\eta_{a}=0.3 / \mathrm{ps}\right.$; green curve) a coupling to the $\mathbf{b}$ mode $\left(\eta_{b}=1.0 / \mathrm{ps}\right.$ and $\left.\eta_{a b}=6.0 / \mathrm{ps}\right)$ calculated using Eqs. (2) and (3) and Eq. (1), respectively. The blue-dashed curve is calculated using Eq. (1) with the $T_{e}$-dependent friction. See the text for the rest of the parameters used herein. 


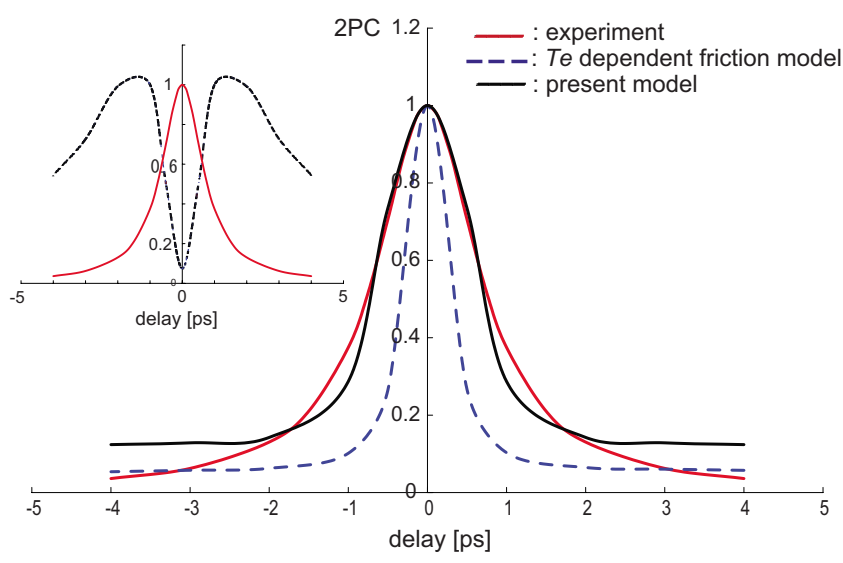

FIG. 3. (Color online). Normalized 2PC for the hopping probability as a function of time delay between two pump pulses. The black curve is calculated using the present model and compared with the $T_{e}$-dependent friction model (blue-dashed curve) and the experimental result (red curve) from Ref. 3. The inset (black-dashed curve) shows $2 \mathrm{PC}$ calculated using constant friction model with $\eta_{a}=0.3 /$ ps. The parameters are the same in Fig. 2 .

$$
R(t)=\eta_{e} \frac{U_{0}}{k_{B} T_{a}(t)} e^{-U_{0} / k_{B} T_{a}(t)} .
$$

The empirical and the generalized friction model showed nearly identical results not only for $T_{a}(t)$ but also for $R(t)$. We have confirmed this and have used Eq. (4) to calculate the $2 \mathrm{PC}$ as well as the fluence $(F)$ dependence of the hopping yield given by $Y(F)=\int R(t, F) d t$.

Figure 3 shows the normalized $2 \mathrm{PC}$ for atomic oxygen hopping as a function of time delay $\left(t_{d}\right)$ between the two pump pulses of the equal fluence. The experimental (red curve) and the calculated results (blue-dashed curve $)^{3}$ using $\eta_{a}\left(T_{e}\right)=\eta a^{0} T_{e}^{2}$ in Eq. (1) are shown for comparison to our numerical result (black curve). In both cases the $2 \mathrm{PC}$ exhibits a peak at $t_{d}=0$, while a dip is observed when we use a constant friction $\eta_{a}=0.3$ / ps in Eq. (1) as shown in the insert (black-dashed curve). Here we used $U_{0}=1.4 \mathrm{eV}$ in Eq. (4) and $0.8 \mathrm{eV}$ in Eq. (5), respectively. In the present theory $T_{a}\left(t_{d}\right)$ shows a peak at $t_{d}=0$ due to the strong vibrationalenergy transfer mediated by the friction coupling to the substrate and bears a close resemblance to $T_{e}\left(t_{d}\right)$. We also found that a peak evolves to a dip in the $2 \mathrm{PC}\left(t_{d}=0\right)$ with a decrease in $\eta_{a b}$. We note that the $2 \mathrm{PC}\left(t_{d}=0\right)$ exhibits a peak even when there is no direct heating of the a mode, i.e., $\eta_{a}=0$, as far as we assume an efficient friction $\eta_{b}$ and mode coupling $\eta_{a b}$. As has been found before ${ }^{15}$ the width of the 2PC becomes narrower with increasing $U_{0}$. In the analysis using $\eta_{a}\left(T_{e}\right)$ the best agreement with the experimental results was achieved for $U_{0}=1.2 \mathrm{eV}$, which is, however, much larger than that estimated for the thermal activated diffusion. Nevertheless, the numerical fitting (blue-dashed curve in Fig. 3) is quite narrow compared to the experimental result (red curve). Our choice of $U_{0}=1.4 \mathrm{eV}$ nicely reproduces the experimental $2 \mathrm{PC}$ except in the tail at longer time delay. This fit using significantly small value of $\eta_{a}$ compared to $\eta_{b}$ convinces us that the frustrated translational mode of the atomic oxygen can be heated up through the mode coupling to the

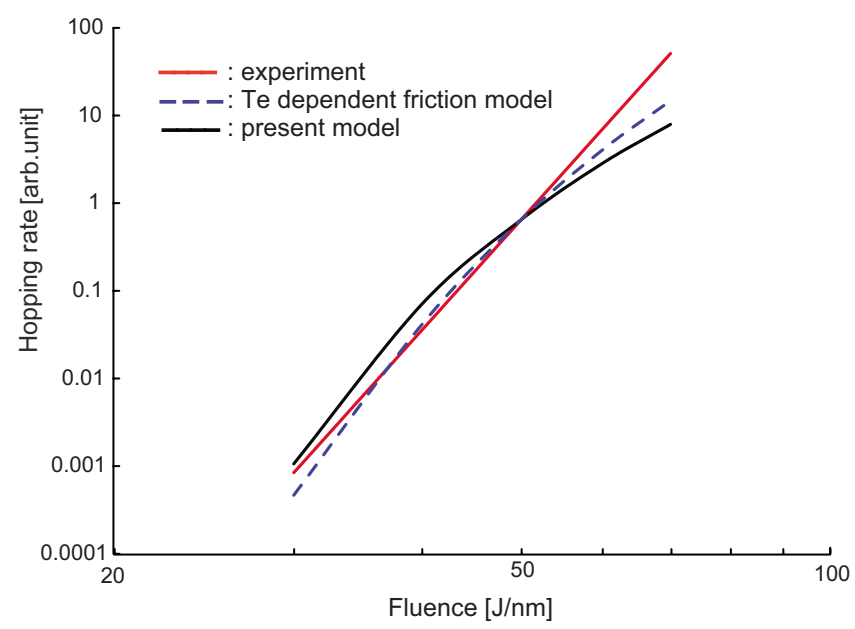

FIG. 4. (Color online). Fluence dependence for hopping probability; the present model (black curve), the $T_{e}$-dependent friction model (blue-dashed curve), and the experimental result (red curve) from Ref. 3. The parameters are the same in Fig. 3.

O-Pt stretch mode with an efficient friction coupling to the hot electrons. The deviation of the fit from the experimental data at the wings, however, indicates a slower cooling rate (higher reaction rate) of $T_{a}(t)[R(t)]$ in the present theory than the actual reaction takes place at long time after laser excitation.

The width of a $2 \mathrm{PC}$ provides information about the timescale of the energy transfer from the initial excitation of the electrons to the adsorbate motion and allows the distinction between an electron- and a phonon-mediated process. ${ }^{1} \mathrm{We}$ obtain the full width at half maximum (FWHM) $\tau=1.44 \mathrm{ps}$ [the $\eta_{a}\left(T_{e}\right)$ model gives $\mathrm{FWHM}=0.68 \mathrm{ps}$ ], which is very close to the experimentally observed FWHM=1.45 ps.

Using $T_{e}(t)$-dependent friction Szymanski et al. ${ }^{7}$ attempted to reproduce the $2 \mathrm{PC}$ for desorption of molecular oxygen from $\operatorname{Pd}(111)$. They found that the reproduction of their experimental $2 \mathrm{PC}$ is significantly improved with the use of a desorption barrier much higher than that determined from thermal desorption experiments. A choice of the activation barrier height is one of the key issues in the analysis of adsorbate dynamics induced by ultrafast laser pulses. In a recent review by Frischkorn ${ }^{16}$ and in Ref. 1 it is pointed out that the multidimensionality of the relevant potential energy surface, which governs the reaction dynamics, is the origin of the discrepancy between the values for $U_{0}$ derived from the friction calculations based on a truncated onedimensional potential and those experimentally obtained from thermally induced reactions. More importantly a fundamental issue is how we can be sure to use Arrhenius type of reaction rate, which assumes a thermal equilibrium of a system, for adsorbate dynamics taking place on ultrafast time scale.

Figure 4 shows the fluence dependence of hopping. Here the experimental (red curve) and numerical results (blue and black curve for the $T_{e}$-dependent friction model and for the present model, respectively) are rescaled and set to 1 at $F$ $=50 \mathrm{~J} / \mathrm{nm}^{2}$. All parameters used here are the same as in the calculation of the 2PC shown in Fig. 3. The reason for the rescaling is that we do not expect a simple one-dimensional 
model to get the absolute numbers correct. ${ }^{17}$ The extremely strong nonlinear fluence dependence $\sim F^{15}$ has been better reproduced by the $T_{e}$-dependent friction model than that calculated by the present model. Nevertheless our model also reproduces an extremely nonlinear fluence dependence although not in a power law. This deviation from the experimental result is not a serious shortcoming at a level of the present modeling. As discussed in Ref. 1, the experimentally observed reaction yield-vs-fluence dependences are well described by a power law $Y=F^{n}$, with $n=3-8$ in a limited fluence range. A nonlinear fluence dependence of the reaction yield, however, does not allow us to draw any conclusion regarding the excitation mechanism. A better agreement with the experimental result can be obtained by varying the parameters $\eta_{a}, \eta_{b}, \eta_{a b}$, and $U_{0}$ within their physically reasonable ranges. However we find that none of them is able to reproduce the $2 \mathrm{PC}$ better than that shown in Fig. 3. We emphasize that our modeling of indirect heating reproduces a peak in the $2 \mathrm{PC}$ and extreme strong fluence dependence of atomic oxygen hopping with the same set of properly chosen parameters.

In summary we have applied the heat transfer equations via a direct friction coupling and an indirect mode-mode coupling to the FT mode responsible for atomic oxygen hopping on a stepped Pt surface. One of the possible elementary processes behind the phenomenological $T_{e}$-dependent friction model used before is an efficient activation of the FT mode via anharmonic coupling to the molecule-surface stretch mode excited by hot electrons. In the present theory the effective friction coupling to the reaction coordinate mode depends on the transient temperature of the partner vibrational mode $T_{b}(t)$, which immediately follows $T_{e}(t)$ in the case of the efficient mode coupling accompanied with a simultaneous excitation and de-excitation of the electronhole pairs in the substrate. The time scales of the heat transfer by the mode coupling and of $1 / \eta_{b}$ need to be shorter than the electron-phonon coupling time, otherwise the hotelectron heat transfers away from the surface into the bulk phonons. We have added an additional coordinate (that of the b mode) to the very simple one-dimensional theory that have been used for the past decades. This captures the most important features of the experimental results, and is helpful in getting a better description of the mechanisms at work. At the present we believe that our modeling of the $T_{b}(t)$-dependent mode coupling provides a possible elementary process behind the empirical $T_{e}(t)$-dependent friction model. The validity of the present theory can be reinforced by direct time-resolved observation, which permits a realtime monitoring of the temperature of the vibrational modes involved in the adsorbate motions induced by femtosecond laser excitation. ${ }^{2}$

We thank M. Bonn for critical reading of the manuscript and valuable comments and U. Höfer for useful discussions. The present work was supported by the Grant-in-Aid for Scientific Research B (No. 18340085) from the Japan Society for the Promotion of Science.
${ }^{1}$ F. Frischkorn and M. Wolf, Chem. Rev. (Washington, D.C.) 106, 4207 (2006).

${ }^{2}$ E. H. G. Backus, A. Eichler, A. W. Kleyn, and M. Bonn, Science 310, 1790 (2005).

${ }^{3}$ K. Stépán, J. Güdde, and U. Höfer, Phys. Rev. Lett. 94, 236103 (2005).

${ }^{4}$ U. Engström and R. Ryberg, Phys. Rev. Lett. 82, 2741 (1999); J. Chem. Phys. 112, 1959 (2000).

${ }^{5}$ T. Komeda, Y. Kim, M. Kawai, B. N. J. Persson, and H. Ueba, Science 295, 2055 (2002).

${ }^{6}$ J. I. Pascual, N. Lorente, Z. Song, H. Conrad, and H.-P. Rust, Nature (London) 423, 525 (2003).

${ }^{7}$ P. Szymanski, A. L. Harris, and N. Camillone III, Surf. Sci. 601, 3335 (2007).

${ }^{8}$ M. Brandbyge, P. Hedegard, T. F. Heinz, J. A. Misewich, and D.
M. Newns, Phys. Rev. B 52, 6042 (1995).

${ }^{9}$ C. Puglia, A. Nilsson, B. Hernnäs, O. Karis, P. Bennich, and N. Martensson, Surf. Sci. 342, 119 (1995).

${ }^{10}$ B. N. J. Persson and J. W. Gadzuk, Surf. Sci. 410, L779 (1998).

${ }^{11}$ B. N. J. Persson and H. Ueba, Phys. Rev. B 76, 125401 (2007).

${ }^{12}$ H. Ueba and B. N. J. Persson, Phys. Rev. B 77, 035413 (2008); J. Phys.: Condens. Matter 20, 224016 (2008).

${ }^{13}$ S. I. Anisimov, B. L. Kapeliovich, and T. L. Perelman, Sov. Phys. JETP 39, 375 (1974).

${ }^{14}$ C. Lei, M. Bauer, K. Read, R. Tobey, T. Popmintchev, M. M. Murnane, and H. C. Kapteyn, Phys. Rev. B 66, 245420 (2002).

${ }^{15}$ J. Güdde and U. Höfer, J. Phys.: Condens. Matter 18, S1409 (2006).

${ }^{16}$ C. Frischkorn, J. Phys.: Condens. Matter 20, 313002 (2008).

${ }^{17} \mathrm{M}$. Bonn (private communication). 